\title{
The Effect of Virtual Reality on Anxiety and Pain in Patients Undergoing Gynecological Surgery (VRAP-G); a Randomized Controlled Trial
}

\section{Kreijveld BJ*, Bekkers IPW, Mulder E and Wassen MMLH \\ Department of Obstetrics and Gynecology, Zuyderland Medical Center, Heerlen, Netherlands}

*Corresponding author: Bob Kreijveld, Department of Obstetrics and Gynecology, Zuyderland Medical Center, Heerlen, Netherlands

Received: December 27, 2020; Accepted: J anuary 21, 2021; Published: J anuary 28, 2021

\begin{abstract}
Background: Lack of postoperative acute pain management is associated with increased morbidity, longer recovery time, more opioid use and subsequently increased health care costs. There is increasing evidence that Virtual Reality (VR) is effective in the reduction of acute pain. Alternative methods to reduce postoperative pain and multimodal analgesia are necessary for acute postoperative pain management and to reduce opioid use and their adverse effects. The aim of this study is to explore the effect of VR on pain in the postoperative period after elective gynecological surgery.
\end{abstract}

Methods and Design: The study concerns a non-blinded, single center, randomized controlled trial. Eligible women fulfilling the inclusion criteria and receive elective gynecological surgery in the Zuyderland Medical Center will be randomized for participation. The study population will be randomly divided into the intervention group (VR-group) or the standard care- group. The intervention group can choose for an immersive guided relaxation VR experience or an interactive VR experience during the pre- and postoperative period additional to the usual standard care. The participants randomized to the standard caregroup will receive only the usual standard care pre-and postoperative.

The primary outcome is postoperative pain measured on a Numeric Rating Scale (NRS). A total of 30 patients have to be included in each group. This means that a total of 60 women will have to be included in this study. Secondary outcomes are; evaluating pre-and postoperative anxiety, pain catastrophizing, analgesic use, length of hospital stay between both groups and to explore tolerability, feasibility and satisfaction of VR use.

Discussion: This study will provide insight as to whether in women who receive gynecological surgery, VR is an effective method to reduce postoperative pain and subsequently opioid use.

Keywords: Virtual Reality; Obstetrics and Gynecology; Anesthesia; Protocol

\section{Introduction}

"An unpleasant sensory and emotional experience associated with actual or potential tissue damage, or described in terms of such damage", is the definition of pain according to the International Association for the Study of Pain (IASP) [1]. Severe post-operative pain is experienced by approximately $50-80 \%$ of the patients $[2,3]$.

Lack of postoperative acute pain management is associated with increased morbidity, longer recovery time, more opioid use and subsequently increased health care costs $[2,4,5]$. Postoperative pain can cause a higher morbidity because of cardiovascular, pulmonic and gastro-intestinal problems [4,5].

Surgery and acute pain are both independent risk factors for the development of chronic pain $[4,6]$. The reduction of acute pain and therefore managing postoperative pain may contribute to the prevention of chronic pain [6]. In general, nowadays, medication is the treatment of choice for acute pain and mainly opioid analgesics are used for pain relief [7]. Worldwide this contributes to excessive drug dependence and opioid abuse. Opioid use comes with several negative side effects, for example a potential delay in recovery and an increased risk of permanent disability [8]. Currently, new methods related to pain management are developed. One of the new methods is Virtual Reality (VR), which is an upcoming technology used within healthcare. It is thought that the perception of pain is related to the amount of attention that is given to pain stimuli [9]. The theory behind the working mechanism of VR to reduce pain is that VR acts as a distraction to limit the user's processing of nociceptive stimuli, by stimulating the visual cortex in the brain [10]. In 2000, Hoffman published the first preliminary evidence for the beneficial effect of VR in reducing pain in a burn care unit [11]. They also showed a trend to experience less anxiety during VR use. In the next upcoming years, a growing body of research was done to explore the effects of VR. A recent systematic review and meta-analysis showed VR to be an effective treatment for reducing acute pain [8]. Next to being effective in reducing pain, it is also a proven useful tool in reducing
Austin J Anesthesia and Analgesia - Volume 9 Issue 1 - 2021 ISSN : 2381-893X | www.austinpublishing group.com Kreijveld et al. (C) All rights are reserved
Citation: Kreijveld BJ, Bekkers IPW, Mulder E and Wassen MMLH. The Effect of Virtual Reality on Anxiety and Pain in Patients Undergoing Gynecological Surgery (VRAP-G); a Randomized Controlled Trial. Austin J Anesthesia and Analgesia. 2021; 9(1): 1095 
preoperative anxiety $[12,13]$. VR can be used as a safe, non-invasive, analgesic method, without risks of drug addiction and minimum side effects $[14,15]$. In view of the worldwide need to reduce opioid use there is need for critical evaluation of alternative methods to decrease postoperative pain. We therefore propose a single center Randomized Controlled Trial (RCT) on this subject.

In this study, we want to explore the effect of VR on postoperative pain in patients undergoing elective gynecological surgery as a serious alternative for pain medication.

\section{Materials and Methods}

\section{Aims}

The aim of this study is to explore the effect of VR on pain in the postoperative period after elective gynecological surgery. We hypothesize VR will reduce post-operative pain and consequently postoperative opioid use and anxiety reduction. The study will also provide insight on whether VR in women scheduled for gynecological surgery will influence pre-operative anxiety, analgesic use, length of hospital stay compared to standard-care.

\section{Study Design, Participants and Outcome Measures}

The design of this study is a single center, RCT. Patients 18 years and older of age, native Dutch speakers, who have an indication for gynecological surgery and who give written and oral informed consent are eligible to participate in this study. Exclusion criteria are chronic pain patients; defined as 'persistent or recurrent pain lasting longer than 3 months' [16], chronical use of pain medication (opioids), history of prior opioid use defined as use within 8 to 90 days prior to the surgical procedure, alcohol or drug abuse, known car sickness, epileptic insults in previous history, psychotically seizures in previous history, claustrophobia, blindness or a history of mental illness. The primary outcome is reduction in postoperative pain score on a Numeric Rating Scale (NRS). Secondary outcomes include pre-operative anxiety using the State-Trait Anxiety Inventory 6 questionnaire (STAI-6), pain catastrophizing using the Pain Catastrophizing Scale questionnaire (PCS), analgesic use (daily use of paracetamol, NSAIDs, opioids), length of hospital stay, tolerability, feasibility and satisfaction of VR use (questionnaire) and preoperative pain score (NRS).

\section{Procedures, Recruitment, Randomization and Collection of Baseline Data}

Patients who are planned for elective gynecological surgery will be informed about the study during their visit at the outpatient clinic. Eligible patients who fulfill the inclusion criteria will be identified and counselled by the research coordinator or staff of the Zuyderland Medical Centre. Before entry into the study, the research coordinator and/or the staff will explain to potential participants the aims, methods, reasonably anticipated benefits, and potential hazards of the study. They will be informed that their participation is voluntary and that they may withdraw consent to participate at any time during the study. They will be informed that choosing not to participate will not affect their care. After giving sufficient information, written informed consent must be obtained. After receiving written informed consent and before randomization, pre-operatively a baseline questionnaire, STAI-6 questionnaire and PCS questionnaire will be filled in preoperatively on the ward. Subsequently the randomization procedure will be proceeded.

Randomization will be conducted by the data management randomization program of Zuyderland Medical Centre. Randomization will be 1:1 for intervention and the standard caregroup. Eligible patients will be randomized to either the VR- group and receive additional VR during the pre- and postoperative period or undergo standard pre- and postoperative management (standard care- group).

At baseline, several questionnaires will be collected. The baseline characteristics will be collected pre-operatively; age, BMI, ethnicity, education level, marital status, vaginal parity, ASA-classification, medication use, intoxications, chronic pain, surgical history, indication of surgery, type of planned surgery. If patients do not want to answer certain questions, for example marital status or education level, they are free to answer 'unknown'. It is possible that these characteristics may be of influence of pain experience or the experiences in VR. These can be confounders in the study.

The STAI-6 questionnaire is a short questionnaire about the feelings of a person in that specific moment [17]. It consists of questions about anxiety, satisfaction and worries. Women were asked to rank on a 4 -point scale ( $0=$ not at all, $4=$ completely) reflecting on the feelings in that specific moment. To calculate the total STAI score, you have to reverse scoring of positive items (calm, relax, content etc.) so $1=4$ points, $2=3$ points, $3=2$ points, $4=1$ points. You can sum all the scores, multiply the total score by 20 and divide this by 6 [17]. The score ranges from 20 to 90 and the higher the score the more anxiety the person experiences. The STAI questionnaire shows the experience of anxiety at baseline.

The PCS has been designed to assess three various dimensions of catastrophizing concerning rumination, magnification and helplessness. It consists of 13 statements describing different feelings and thoughts that can be experienced when in pain. Women were asked to rank on a five-point scale $(0=$ not at all; $4=$ all the time) reflecting on a painful experience in the past [18]. The subscales of the PCS can be calculated separately or overall, with a maximum total score of $52[18,19]$. The higher the score, the more catastrophizing thoughts are present (full questionnaires regarding this research can be send in request). The PCS shows how people are able to cope with pain and various thoughts.

\section{Interventions}

VR- group: The participants randomized into the VR group can choose for an immersive guided relaxation VR experience or an interactive VR experience. We will register which option the patients choose. Pre-operatively on the gynecological ward, the level of anxiety and pain is scored using the NRS. After this, the VR-group will receive their VR experience of own choice on the pre-operative anesthetic room during 10-15 minutes. Directly after this VR intervention, the level of anxiety and pain is scored from 0-10.

In the postoperative setting the patients will receive a total of 3 VR-interventions of choice a day, during 10-15 minutes on the first and second day postoperative. The mean pain score (NRS) from 0-10 will be registered during 24 hours for day 1 and day 2 postoperative. The VR intervention is additional to the standard local hospital postoperative care management and the standard pain protocol. The 


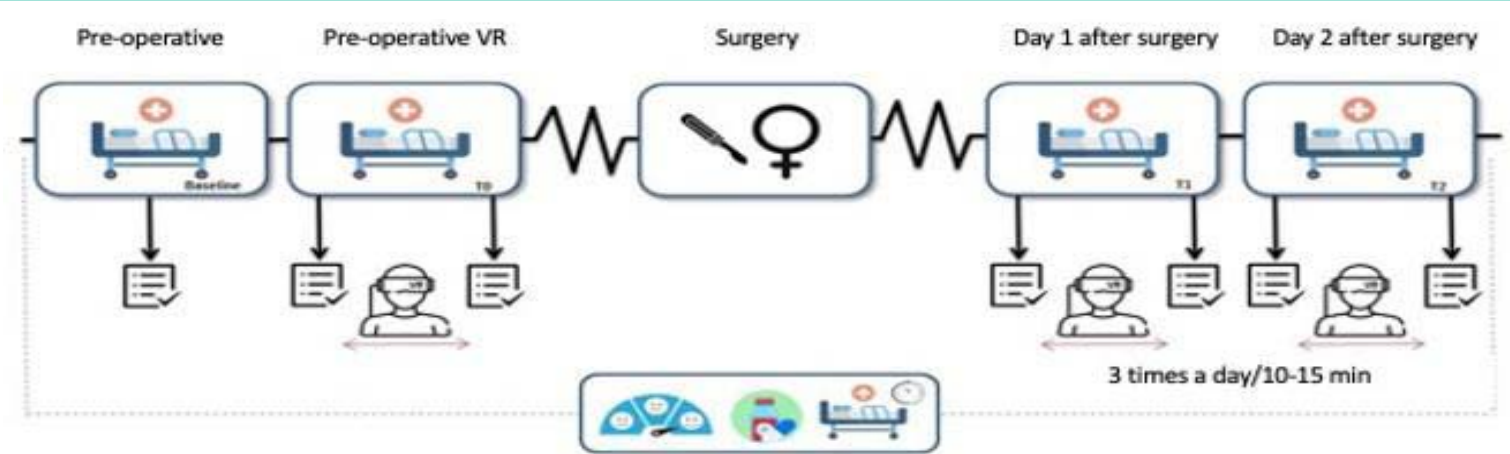

Figure: The VR-intervention will be given using the VR Pico G2 4K Enterprise glasses with touchpad

VR-intervention will be given using the VR Pico G2 4K Enterprise glasses with touchpad (Figure).

Standard- Care Group: The participants randomized into the standard care-group will receive the usual standard pre-and postoperative management. Pre-operatively on the gynecological ward, the level of anxiety and pain is scored from 0-10 (NRS). After 10-15 minutes the level of pain and anxiety is again scored from a scale from $0-10$. The mean pain score (NRS) from $0-10$ will be registered during the first day and the second day postoperative.

\section{Statistical Issues}

Sample Size: We are planning a study of independent cases and controls with 1 control(s) per case. Our hypothesis is that VR (intervention) will significantly improve the mean NRS for pain after surgery with $15 \mathrm{~mm}$ with a standard deviation of $20 \mathrm{~mm}$.

On the assumption of an effect of $15 \mathrm{~mm}$ we would need 26 patients per group: 30 taking into account loss of follow-up. Thus 60 patients need to be included. With this sample size we are able to achieve a power of 0.8 . The Type I error probability associated with this test of this null hypothesis is 0.05 .

Data Analysis: The data will be analyzed using the program SPSS (version 26). A Chi-square test will be used to assess categorical variables and the independent sample $\mathrm{T}$-test will be used for continuous variables in case of normal distribution based on the skewness, kurtosis, and graphical representation (histogram) of the continuous variables. The mean NRS-score with standard deviation will be calculated for pain as well as anxiety in both groups, when the data is normally divided. When the data is not normally divided, then a median with interquartile range will be calculated. For categorical variables, frequency and percentage will be calculated. Depending on the number of missing values, the missing values will be excluded or imputed. This depends on the number of missing values.

The differences in postoperative NRS-scores of pain in between both groups (VR-intervention and standard-care group) will be analyzed using the linear mixed models analysis. The NRS-score of pain of the first and second day postoperative will be calculated into means with standard deviation. The NRS-score of pain of the VR group and standard-care group will be compared to see whether VR influences the pain experience. Different variables, like age, BMI, ethnicity, education level, marital status, vaginal parity, ASAclassification, medication use, intoxications, chronic pain, surgical history, indication of surgery, type of planned surgery, will be considered in the statistical analysis. These are taken into account because these variables can be possible confounders in the results of the study.

The differences in NRS-scores preoperative- and postoperative of anxiety in both groups (VR-intervention and standard-care group) will be analyzed using the linear mixed models' analysis. The results of the STAI-questionnaire, PCS questionnaire, analgesic use and length of hospital stay will be calculated into means with standard deviation and confidence interval. We want to compare the baseline characteristics and questionnaires (STAI and PCS) to see whether the groups are equally divided. The STAI and PCS questionnaire are continuous variables, we will use the independent sample t-test. The tolerability, feasibility and satisfaction of VR use will be calculated into means and frequencies.

\section{Ethical Consideration}

This study has been approved by the ethics committee of the Zuyderland Medical Center (METC. NL72290.096.20).

\section{Discussion}

Increasing use and abuse of opioids is a global and worldwide problem. To manage this opioid epidemy, we have to explore alternative methods to reduce acute pain postoperatively. The reduction of acute pain will reduce the development of chronic pain. This research will contribute to the development effective and safer opioid- sparing alternative interventions for pain.

\section{References}

1. Treede RD. The International Association for the Study of Pain definition of pain: as valid in 2018 as in 1979, but in need of regularly updated footnotes. Pain Rep. 2018; 3: e643.

2. Popping DM, Zahn PK, Van Aken HK, Dasch B, Boche R, Pogatzki-Zahn EM Effectiveness and safety of postoperative pain management: a survey of 18 925 consecutive patients between 1998 and 2006 ( $2^{\text {nd }}$ revision): a database analysis of prospectively raised data. Br J Anaesth. 2008; 101: 832-840.

3. Rizalar S, Baltaci N. Kahraman S. Investigation of post-operative pain levels and nursing inverventions following gynecologic surgery. Nursing Practice Today. 2015

4. Gan TJ. Poorly controlled postoperative pain: prevalence, consequences, and prevention. J Pain Res. 2017; 10: 2287-2298.

5. Joshi GP, Ogunnaike BO. Consequences of inadequate postoperative pain relief and chronic persistent postoperative pain. Anesthesiol Clin North Am. 2005; 23: 21-36. 
6. Mills SEE, Nicolson KP, Smith $\mathrm{BH}$. Chronic pain: a review of its epidemiology and associated factors in population-based studies. Br J Anaesth. 2019; 123 e273-e283.

7. Zhao S, Chen F, Feng A, Han W, Zhang Y. Risk Factors and Prevention Strategies for Postoperative Opioid Abuse. Pain Res Manag. 2019; 2019: 7490801.

8. Mallari B, Spaeth EK, Goh H, Boyd BS. Virtual reality as an analgesic for acute and chronic pain in adults: a systematic review and meta-analysis. J Pain Res. 2019; 12: 2053-2085.

9. Botella C, Banos AGPR, Quero S, Breton-Lopez J. Virtual Reality in the treatment of pain. Journal of Cyber Therapy and Rehabilitation. 2008; 1.

10. Spiegel B, Fuller G, Lopez M, Dupuy T, Noah B, Howard A, et al. Virtual reality for management of pain in hospitalized patients: A randomized comparative effectiveness trial. PLoS One. 2019; 14: e0219115.

11. Hoffman HG, Patterson DR, Carrougher GJ. Use of virtual reality for adjunctive treatment of adult burn pain during physical therapy: a controlled study. Clin J Pain. 2000. 16: 244-250.

12. Robertson RK, Fick D. The effect of virtual reality in reducing preoperative anxiety in patients prior to arthroscopic knee surgery: A randomsed controlled trial. 2017.
13. Dehghan $F$, Jalali $R$, Bashiri $H$. The effect of virtual reality technology on preoperative anxiety in children: a Solomon four-group randomized clinical trial. Perioper Med (Lond). 2019; 8: 5.

14. JahaniShoorab N, Zagami SE, Nahvi A, Mazluom SR, Golmakani N, Talebi $M$, et al. The Effect of Virtual Reality on Pain in Primiparity Women during Episiotomy Repair: A Randomize Clinical Trial. Iran J Med Sci. 2015; 40: 219-224.

15. Li A, Montano Z, Chen VJ, Gold JI. Virtual reality and pain management: current trends and future directions. Pain Manag. 2011; 1: 147-157.

16. Treede RD, Rief W, Barke A, Aziz Q, Bennett MI, Benoliel R, et al. A classification of chronic pain for ICD-11. Pain. 2015; 156: 1003-1007.

17. Marteau TM, Bekker $\mathrm{H}$, The development of a six-item short-form of the state scale of the Spielberger State-Trait Anxiety Inventory (STAI). Br J Clin Psychol. 1992; 31: 301-306.

18. Michael JL, Sullivan P. The Pain Catastrophizing Scale-User Manual. 2009.

19. Sullivan MJLB, Pivik SRJ. The Pain Catastrophizing Scale: Development and Validation. Psychological Assessment. 1995. 